Qiang Li, Valentin L. Popov

\title{
A numerical study of JKR-type adhesive contact of ellipsoids
}

Journal article | Accepted manuscript (Postprint)

This version is available at https://doi.org/10.14279/depositonce-10122

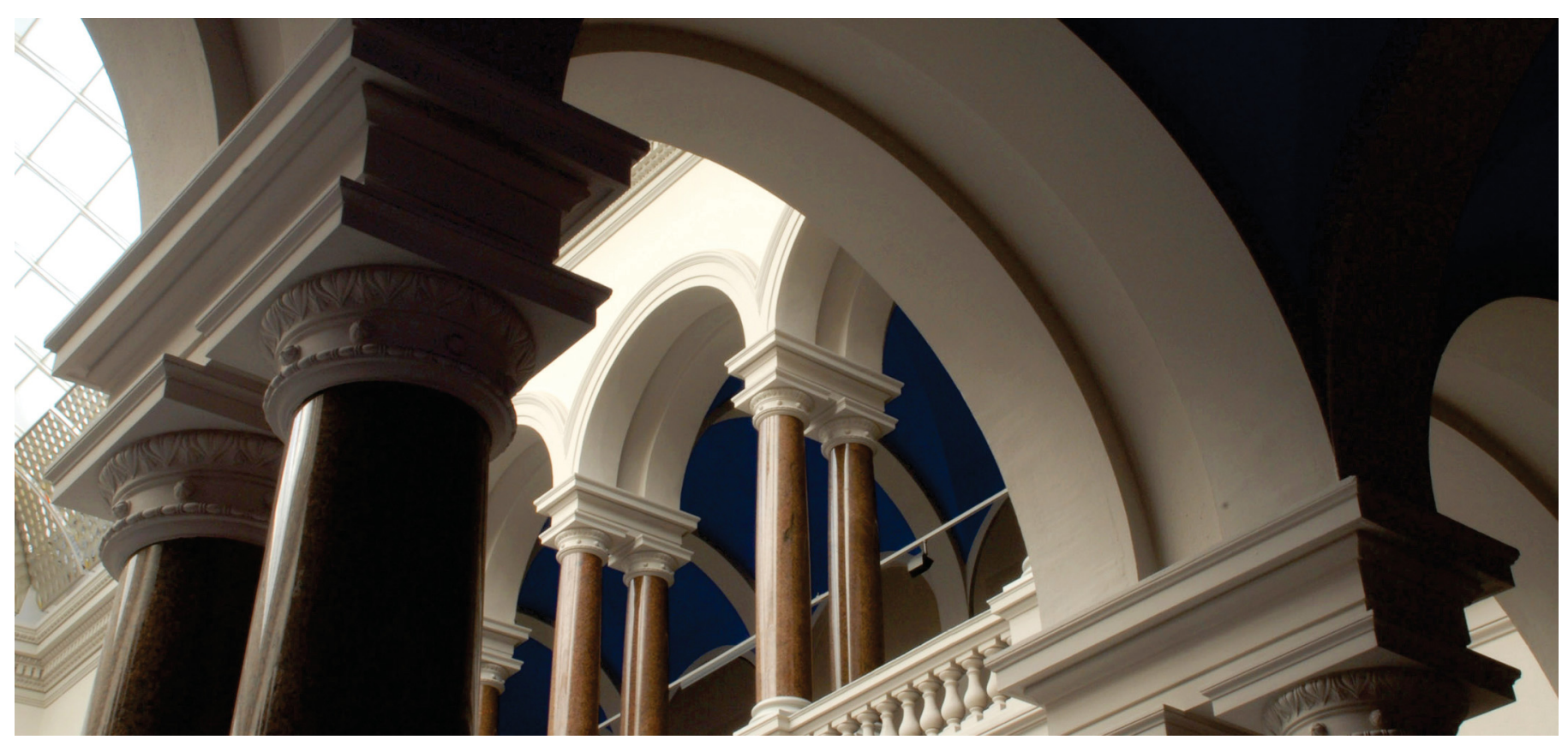

Li, Q., \& Popov, V. L. (2020). A numerical study of JKR-type adhesive contact of ellipsoids. Journal of

Physics D: Applied Physics. https://doi.org/10.1088/1361-6463/ab85e9 


\title{
A numerical study of JKR-type adhesive contact of ellipsoids
}

\author{
Qiang $\mathrm{Li}^{1}$, and Valentin L. Popov ${ }^{1,2,3}$ \\ ${ }^{1}$ Institute of Mechanics, Berlin University of Technology, Berlin, Germany \\ ${ }^{2}$ National Research Tomsk State University, Tomsk, Russia \\ ${ }^{3}$ Institute of Strength Physics and Materials Science, Russian Academy of Sciences, Tomsk, Russia \\ E-mail: qiang.li@tu-berlin.de; v.popov@tu-berlin.de
}

\begin{abstract}
Approximate solution by Johnson and Greenwood (2005) for an adhesive contact of an ellipsoid and an elastic half-space is revisited numerically using the FFT-based Boundary Element Method. While for moderate values of the ratio of principal radii of the ellipsoid, $R_{1} / R_{2}$, predictions of the Johnson-Greenwood approximate theory are very good, they become increasingly inaccurate for large values of this parameter. On the basis of numerical simulations, we provide analytical expressions for the dependencies between load, approach and contact area and compare the exact shape of the contact area with the elliptical one assumed in the Johnson-Greenwood-theory.
\end{abstract}

Keywords: Adhesion, elliptical contact, JKR approximation, Boundary Element Method

\section{Introduction}

1971, Johnson, Kendall and Roberts proposed a theory of adhesive contacts of parabolic bodies which is known as JKR theory [1]. While Johnson, Kendall and Roberts derived their theory only for axis-symmetric parabolic bodies, the principle of energy balance which this theory is based on can be applied to an arbitrary contact. The main physical assumption of the JKR theory in that the range of action of adhesive forces is infinitely small (practically meaning smaller than any other length parameter of the system). The opposite case of large range of adhesive interactions was suggested by Derjaguin et al. in 1975 (DMT theory) [2]. The transition between these limiting cases was followed by Tabor who 1977 introduced a parameter determining transition between two limiting cases, which we now call "Tabor parameter" [3]. This transition was illustrated 1992 by Maugis [4], who used a simple model interaction potential. In the present paper we will call all adhesive theories using the assumption of infinitesimally small adhesive range as "JKR-type".

For adhesive contact of an ellipsoid with elastic half space, Johnson and Greenwood proposed an approximate solution which main basic assumptions were: 1 ) the contact area in the adhesive contact remains elliptical and 2) the stress intensity factor (SIF) is equal to the critical value around the periphery (Johnson and Greenwood (2005)) [5]. Already Johnson and Greenwood argued that the contact area is in reality not exactly elliptical. Later, an experimental analysis of contact between two non-parallelly placed cylinders has proven that the contact shape deviates from the elliptical shape, especially at the tip of major axis when the shape is slim [6]. Recently a double-Hertz model was extended to obtain a solution for elliptical contact [7], however is was argued by Greenwood, one of the authors of the original Double-Hertz model [8], that the assumptions of the derivation for adhesive contact in [7] are not correct.

Numerically the elliptic adhesive contact has been studied in the recent years, where surface interaction is assumed to be governed by the Lennard-Jones potential in the simulation [9][10]. These numerical results shown also that the shape of contact area is non-elliptical as observed in experiments in study [6]. Recently a Fast Fourier Transform (FFT)-based Boundary Element Method (BEM) was developed for adhesive contact where the Griffith's criterion of energy balance was applied to find a stress criterion for detachment in pull-off [11]. This is the same physical principle as applied in the classical JKR theory, thus the method reproduces the 
JKR-results numerically with a very high accuracy. The adhesive BEM has been applied to adhesive contact of various indenters including torus [12], elliptical punch and a system of two punches [13]. In all cases where rigorous analytical results are available, they coincide with high precision with the results of numerical simulation using the present version of BEM. An extensive validation by changing mesh orientation, size and so on as well as comparison with specially designed experiments with flat-ended punches having complex shape can be found in [14].

In the present paper we revisit the Johnson and Greenwood (2005) problem of an adhesive ellipsoidal contact using the numerical method mentioned above. In the next Section, we briefly introduce the main findings of Johnson and Greenwood as reference for comparison with numerical results. Section 3 is devoted to a description of the numerical method used in this paper. Section 4 presents the numerical results and comparison with the JG solution. Section 5 contains a general discussion of the findings of the paper. Section 6 concludes the paper.

\section{Johnson-Greenwood (JG) solution of 2005}

We consider the following contact. An ellipsoid having the following surface profile:

$$
h(x, y)=\frac{x^{2}}{2 R_{1}}+\frac{y^{2}}{2 R_{2}},
$$

where $R_{1}$ and $R_{2}$ are principle radii of curvature $\left(R_{1}>R_{2}\right)$, is pressed into an elastic half space with elastic modulus $E$ and Poisson's ratio $v$ with the normal load $F$. Considering adhesion, the parameter $\Delta \gamma$ - the work of adhesion per unit area is involved. Johnson and Greenwood assumed that the contact area has an elliptical shape with the major and minor axes $a$ and $b(a \geq b)$ [5], as shown in Figure 1 .

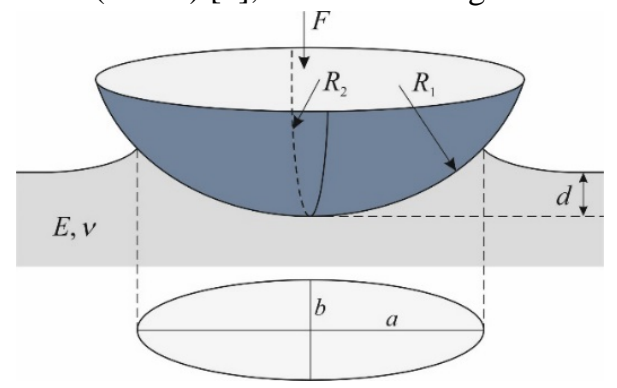

Figure 1 Adhesive contact between a curved surface with two different principal radii of curvature and an elastic half-space. The contact area in JG approximation is assumed to be elliptical.

The JG analytical approximation gives the solution of normal load and indentation depth in dimensionless form [5][6]

$$
\bar{F}=\frac{8}{3 \pi} \frac{g(1-\sqrt{g})^{2}}{\left(\bar{\beta} g^{2}-\bar{\alpha}\right)^{2}}\left[\frac{\bar{\alpha}-\bar{\beta} g^{5 / 2}}{1-\sqrt{g}}-\frac{1}{3}\left(\bar{\beta} g^{2}+\bar{\alpha}\right)\right],
$$

$$
\begin{aligned}
\bar{d}= & \left(\frac{2^{7 / 2}}{9 \pi^{2}}\right)^{2 / 3}\left[\frac{\sqrt{g}(1-\sqrt{g})}{\bar{\beta} g^{2}-\bar{\alpha}}\right]^{4 / 3} \\
& \cdot\left[2 K_{1} \frac{\bar{\alpha}-\bar{\beta} g^{5 / 2}}{1-\sqrt{g}}-\bar{\alpha} B-g^{2} \bar{\beta} D\right]
\end{aligned}
$$

with

$$
\begin{aligned}
& \bar{\beta}=\frac{1}{\lambda} \cdot \frac{\left(\lambda^{2}+1\right) C+D}{(C+D)\left(B+g^{2} C\right)-g^{2} C^{2}}, \\
& \bar{\alpha}=\frac{\lambda+\lambda^{-1}-\bar{\beta} B}{D},
\end{aligned}
$$

and the following definitions of dimensionless force and indentation:

$$
\bar{F}=\frac{F}{3 \pi R_{e} \Delta \gamma}, \bar{d}=d \cdot\left(\frac{4 E^{*}}{9 \pi \Delta \gamma \sqrt{R_{e}}}\right)^{2 / 3},
$$

where $\lambda=\sqrt{R_{2} / R_{1}}, R_{e}=\sqrt{R_{1} R_{2}}, g$ is the axes ratio $g=b / a$ and $K_{1}, K_{2}$ are complete elliptic integrals of the first and second kind respectively (which depend only on the eccentricity $\left.e=\sqrt{1-b^{2} / a^{2}}\right), \quad D=\left(K_{1}-K_{2}\right) / e^{2}$, $B=K_{1}-D, \quad C=(D-B) / e^{2} ; E^{*}$ is effective elastic modulus $E^{*}=E /\left(1-v^{2}\right)$ (assuming the indenter to be rigid). The mean contact radius $c$ defined as $c=\sqrt{a b}$ is also an important parameter for characterization of contact area, the corresponding dimensionless form of it is defined as $\bar{c}=c / c_{r}$ with $c_{r}=\left(9 \pi R_{e}^{2} \Delta \gamma /\left(4 E^{*}\right)\right)^{1 / 3}$. In the following sections, the force, distance and contact radius from numerical results are normalized in the same way as described above, and are compared with this theoretical solution (2). For the circular case with $R_{1}=R_{2}$, the Eq.(2) will become the classic JKR solution for spherical indenter (1971) [1], and this case is denoted as "JKR solution" in the following figures.

\section{Methods}

Since 1990s boundary element formulation has been widely used for solution of contact problems, e.g. in elastohydrodynamic lubrication [15]. It is based on the fundamental solution providing the surface deformation $u(x, y)$ at position $(x, y)$ under a concentrated force acting at the point $(0,0)$ which was found by Boussinesq in 1885 . Using the fundamental solution and the superposition principle, one can easily write the displacement of any point of the surface under the action of an arbitrary distributed stress $p\left(x^{\prime}, y^{\prime}\right)$ : 


$$
u(x, y)=\frac{1}{\pi E^{*}} \iint_{A} \frac{p\left(x^{\prime}, y^{\prime}\right)}{\sqrt{\left(x-x^{\prime}\right)^{2}+\left(y-y^{\prime}\right)^{2}}} \mathrm{~d} x^{\prime} \mathrm{d} y^{\prime} .
$$

In a discrete form by assuming the constant stress on a single rectangular discretization element, it can be written in the form

$$
u_{i j}=K_{i j i^{\prime} j^{\prime}} p_{i^{\prime} j^{\prime}} \text {, }
$$

with the coefficient matrix $K_{i j i^{\prime} j^{\prime}}$ found and documented in many sources, e.g. in [16]. However, Eq. (4) cannot be used for practical calculations as the matrix $K_{i j i^{\prime} j^{\prime}}$ has $N^{4}$ elements (for a simulation with $N \times N$ grid points). To accelerate the evaluation, the convolution technique based on the Fast Fourier Transformation (FFT) is used, which reduces the complexity of the problem from $o\left(N^{4}\right)$ to $o\left(N^{2} \log N\right)$. The BEM simulation is an iterative procedure while each step consists of two sub steps: determining the deformation from the given stress distribution. This step is carried out with a double FFT:

$$
\mathbf{u}=\operatorname{iFFT}[\operatorname{FFT}(\mathbf{K}) \cdot \operatorname{FFT}(\mathbf{p})] .
$$

For the inverse problem, finding the stress from the changed displacement field (after considering the boundary conditions), the conjugate-gradient (CG) method is used. This basic version of BEM is well known in application to contact problems, see e.g. [17][18].

In this paper we study the JKR-type adhesion, so a further developed BEM for JKR-type adhesive contact suggested in [11] is used. The only difference with the non-adhesive version is in the introducing a criterion for detaching of an element. This criterion is exactly the Griffith's criterion based on the energy balance: Considering a single grid element in contact, it is assumed that it will detach if the elastic energy $U_{e l}$ which is released due to detachment exceeds the work of adhesion necessary for generation of the free surfaces, $U_{\text {surf }}$. Elastic energy can be calculated by $U_{e l}=\frac{1}{2} \iint_{A} p u \mathrm{~d} A$, where $A$ is the element area, and surface energy is simply obtained by $U_{\text {surf }}=A \Delta \gamma$. Equating them results in the tensile stress criterion $\sigma_{\text {crit }}$ (the derivation can be found in [11]).

In a numerical procedure, it is realized in the following way. Non-adhesive contact is initially solved by the basic BEM as described above. Now we pull off the indenter with a small incremental indentation step. Firstly, we keep the contact area unchanged then the stresses are calculated by the CG method. The generated tensile stresses are compared with the critical detachment stress. Then with the new contact area this procedure is repeated until a correct contact area is found while all the tensile stresses satisfy this condition. Then the simulation can come to the next pull-off step. The details of this approach including the basic principles, the algorithm and simulation examples can be found in [11].

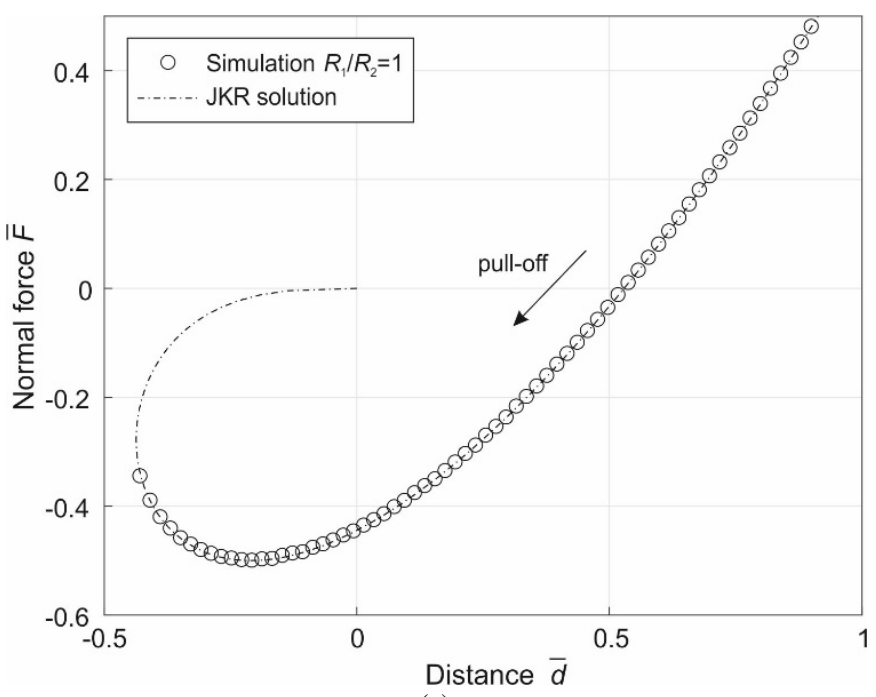

(a)

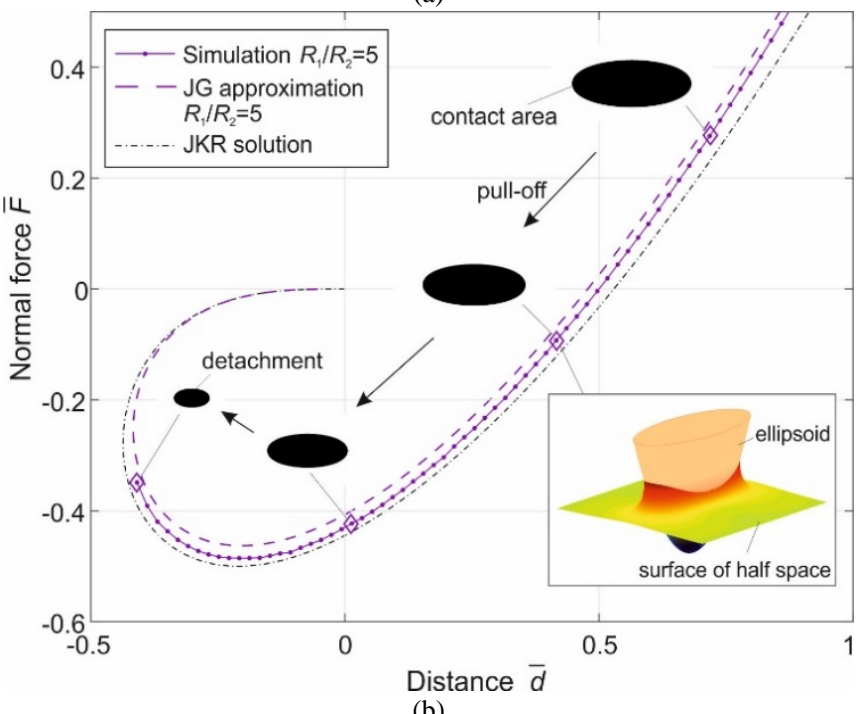

Figure 2 (a) Force-distance relation from pull-off simulation for circular case with $R_{1} / R_{2}=1$. The circles are numerical results and dash-dotted line the theoretical JKR solution. (b) pull-off simulation for ellipsoid with $R_{1} / R_{2}=5$ : force-distance relation, and contact areas in four marked states as well as one contact configuration. Dashed line is the JG solution for $R_{1} / R_{2}=5$.

For illustration, Figure 2 shows two examples of the simulated detachment process for circular case with $R_{1} / R_{2}=1$ (Figure 2a) and for an ellipsoid with $R_{1} / R_{2}=5$ (Figure 2b) under displacement-controlled conditions. From Figure 2a one can see that the numerical simulation (circles) for spherical indenter reproduces the JKR solution (dashdotted line) with high accuracy. In Figure $2 \mathrm{~b}$ for ellipsoid contact areas in the four contact states marked on the curve are also presented, as well as one contact configuration where the geometry of rigid ellipsoid and the deformation of surface of elastic half space can be seen. The JG solution for $R_{1} / R_{2}=5$ and JKR solution are also presented for comparison. These simulations have been carried out on a grid with $2048 \times 2048$ points. 
The results are described in detail in the following section.

\section{Results}
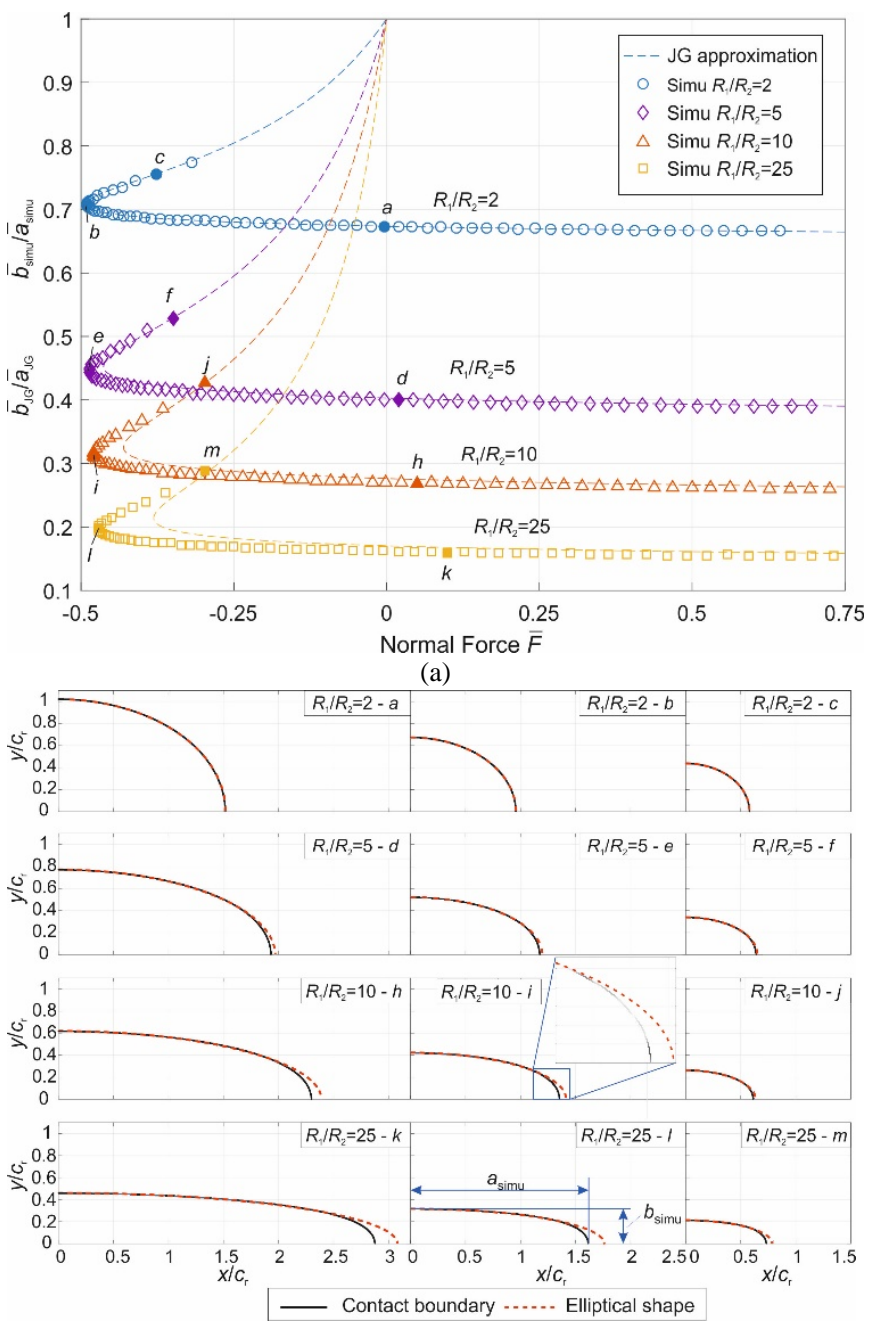

(b)

Figure 3 (a) dependence of ratio $b / a$ and $b_{\text {simu }} / a_{\text {simu }}$ on the normal load for different $R_{1} / R_{2}$. The dashed lines are JG-solutions. (b) Shape of contact areas during the pull-off corresponding to the position $a-m$ marked in (a).

Shape of contact area. Figure 3a shows the dependence of the axes ratio $\bar{b}_{\text {simu }} / \bar{a}_{\text {simu }}$ on the normal load for four different ratios of principle radii of ellipsoid curvature $R_{1} / R_{2}=2,5,10,25$, where $a_{\text {simu }}$ and $b_{\text {simu }}$ are defined as the half-length of contact boundary line in the directions of maximum and minimum cross-section, as illustrated in subplot of Figure 3b. These "half-axes" are represented in the normalized form, $\bar{b}_{\text {simu }}=b_{\text {simu }} / c_{r}$ and $\bar{a}_{\text {simu }}=a_{\text {simu }} / c_{r}$. It is seen that the numerical result on $\bar{b}_{\text {simu }} / \bar{a}_{\text {simu }}-\bar{F}$ curve for the small $R_{1} / R_{2}=2$, follows precisely the JG-solution (solid line). For $R_{1} / R_{2}=5$, the first deviation from the JG-solution is observed but it remains rather small. However, for the large values of $R_{1} / R_{2}=10$ or 25 the deviation becomes essential.

Figure $3 \mathrm{~b}$ shows a series of contact shapes (black solid lines) during the pull-off compared with the exact elliptical shape (red dashed lines). Due to the symmetrical contact, only one quarter shape of the contact border is presented. It can be observed that the contact shape is very close to ellipse for the small $R_{1} / R_{2}=2$ (Figure 3b, $a-c$ ). In other cases (Figure 3b, $d$ $m$ ), there is some deviation from the elliptical shape (the tips are blunter than for an ellipse (red dashed line)). The deviation is larger for slimmer ellipsoids.

Load-distance and load-mean contact radius relation. First, the mean contact radius should be defined. In the JGapproximation, the mean contact radius is defined as $c=\sqrt{a b}=\sqrt{A / \pi}$ where $A$ is contact area. In the numerical simulation define it also over the contact area: $c=\sqrt{A / \pi}$, however, due to the non-elliptical shape of contact area, now $c \neq \sqrt{a b}$.

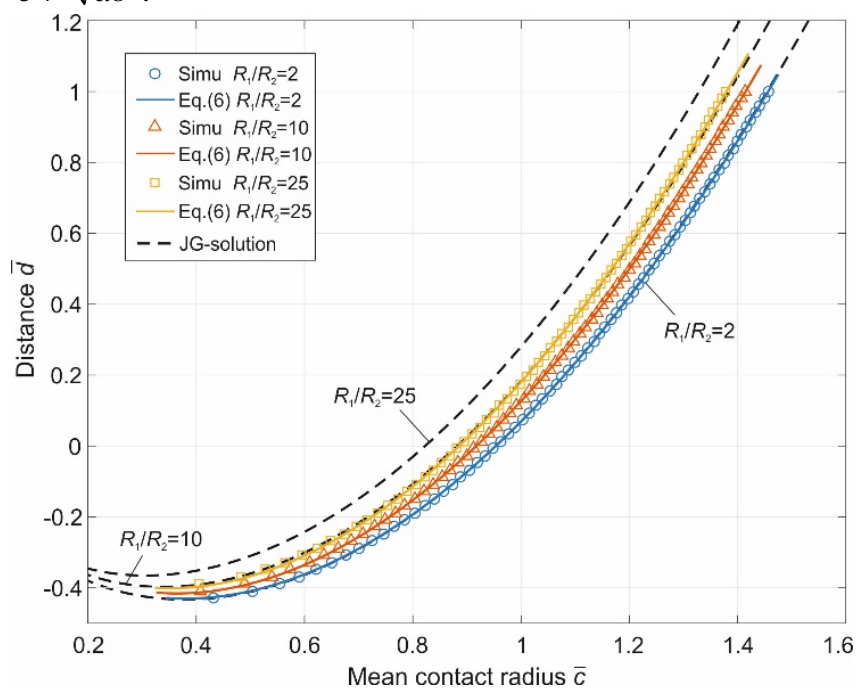

(a)

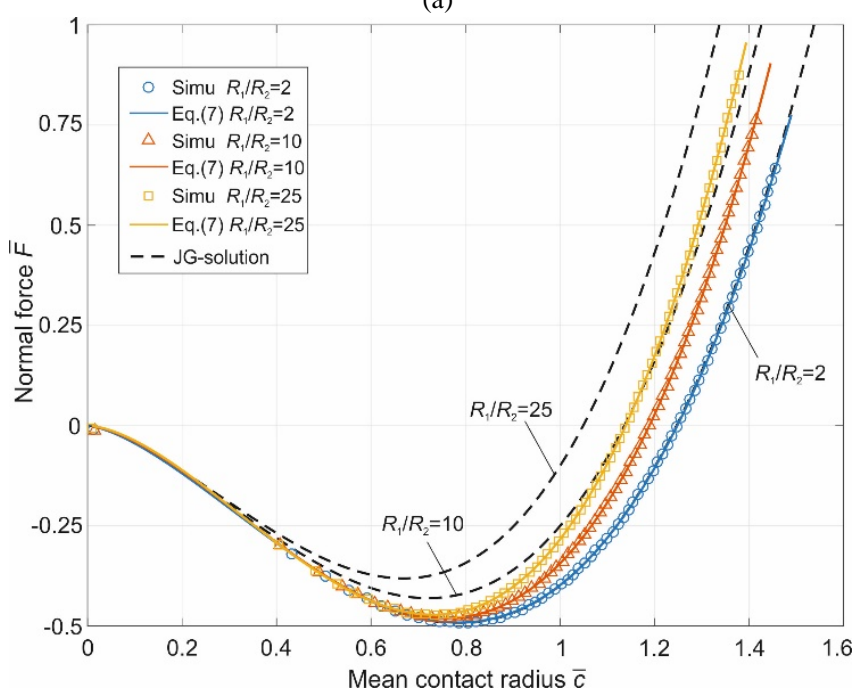

(b) 


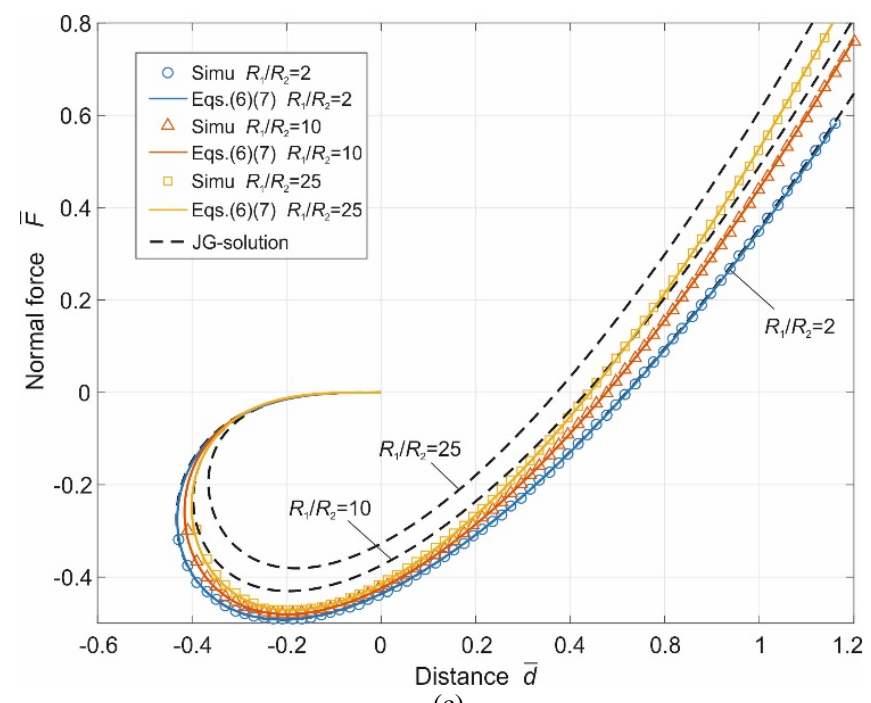

(c)

Figure 4 (a) distance-mean contact radius dependences (b) loadmean contact radius dependences (c) load-distance dependences for three different values of $R_{1} / R_{2}$. Symbols are numerical results, dashed lines the JG-solutions, and the solid lines the new proposed solution (6)(7).

Numerical results for load-contact radius, distance-mean contact radius and load-distance relation are shown in Figure 4a,b,c respectively (symbols) in comparison with the JGapproximation (black dashed lines). Again, one can see that the JG-solution shows good agreement with numerical solution only for the smallest of the three considered ratios $R_{1} / R_{2}=2,10,25$.

Similar to the JKR-solution, we propose analytical expressions for distance-mean contact radius relation and force-mean contact radius relation in the following and the corresponding coefficients are found by fitting these curves: the relation of distance and mean contact radius is

$$
\bar{d}=A \cdot \bar{C}^{2}+B \cdot \bar{C}^{0.5},
$$

with coefficient $A$ and $B$ as a function of $R_{1} / R_{2}$

$$
\begin{gathered}
A=0.05548 \cdot\left(R_{1} / R_{2}\right)^{0.3103}+0.9323 \\
B=0.00658 \cdot\left(R_{1} / R_{2}\right)^{0.5779}-0.9439 .
\end{gathered}
$$

Note that in the JKR-theory for a circular contact it is $A=1$ and $B=-2 / 3 \cdot \sqrt{2}$. The fitting curves with these coefficients can be found in Figure 4a (solid lines). The coefficients $A$ and $B$ and their dependencies on ratio $R_{1} / R_{2}$ are shown in Figure 5a.

The dependence of normal force on the mean contact radius is given by

$$
\bar{F}=c_{1} \cdot \bar{c}^{3}+c_{2} \cdot \bar{C}^{\alpha},
$$

with coefficients $c_{1}, c_{2}$ and $\alpha$ as a function of $R_{1} / R_{2}$

$$
\begin{aligned}
& c_{1}=0.1467 \cdot\left(R_{1} / R_{2}\right)^{0.4872}+0.7893, \\
& c_{2}=-0.1188 \cdot\left(R_{1} / R_{2}\right)^{0.474}-1.23,
\end{aligned}
$$

$$
\alpha=0.1741 \cdot\left(R_{1} / R_{2}\right)^{0.2446}+1.281 .
$$

In the JKR-theory, for a circular contact, $c_{1}=1, c_{2}=-\sqrt{2}$ and $\alpha=3 / 2$. The fitting curves can be found in Figure $4 \mathrm{~b}$ (solid lines). The coefficients $c_{1}, c_{2}$ and $\alpha$ and their dependencies on ratio $R_{1} / R_{2}$ are shown in Figure $5 \mathrm{~b}$.

With the Eqs. (6a) and (7a) we plot the force-distance curve, as shown in Figure 4c (solid lines). One can see that theses analytical approximations describe the obtained numerical results very well.

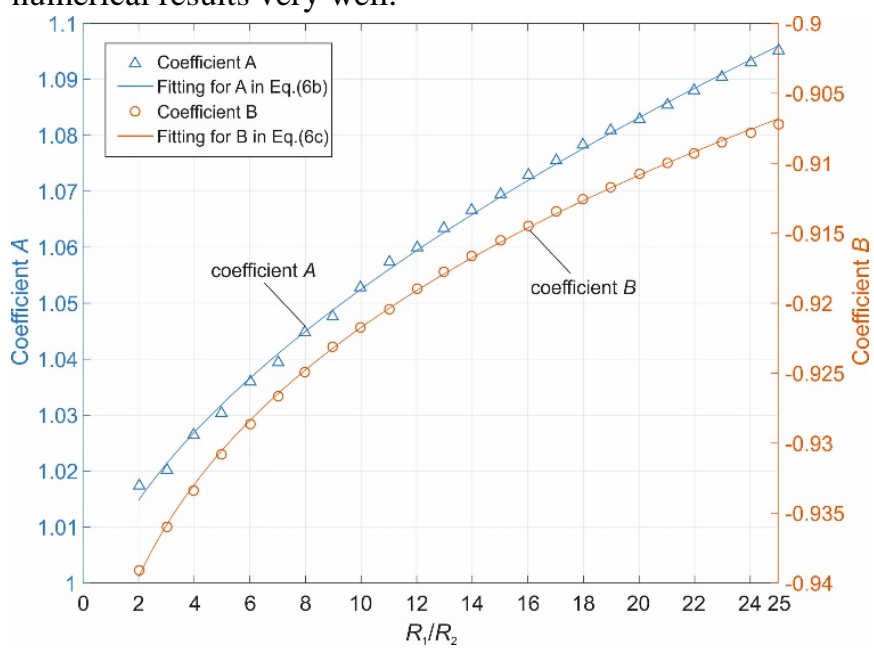

(a)

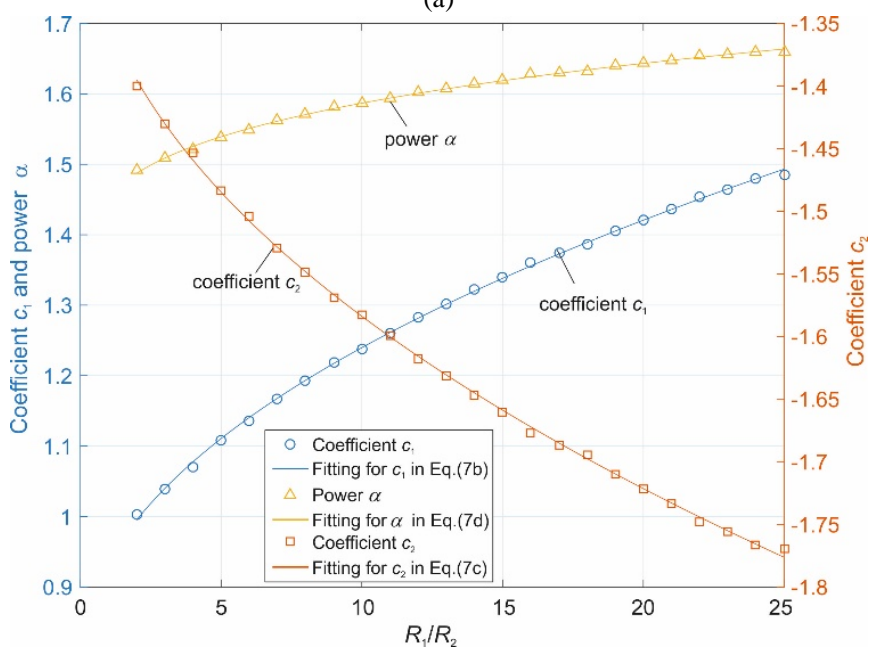

(b)

Figure 5 (a) Coefficients $A$ and $B$ and (b) Coefficients $c_{1}, c_{2}$ and $\alpha$ for different values of $R_{1} / R_{2}$ obtained by fitting the numerical results (symbols), and its analytical expressions Eqs. (6b)(6c) and (7b)-(7d) (solid lines);

Adhesive force. The numerical results show that the normalized adhesive force (absolute value of minimal normal force) decreases with the ratio $R_{1} / R_{2}$, but not so strongly as predicted by JG-solution (Figure 3 and Figure 4). 
These adhesive forces for values of $R_{1} / R_{2}$ from 2 to 50 are shown in Figure 6 (symbols). The numerical results can be described by empirical equation

$$
\bar{F}_{a d}=-0.08429 \cdot\left(R_{1} / R_{2}\right)^{0.08278}+0.5819
$$

or, in dimensional form,

$$
F_{a d}=\left[-0.08429 \cdot\left(R_{1} / R_{2}\right)^{0.08278}+0.58193\right] \cdot 3 \pi \sqrt{R_{1} R_{2}} \Delta \gamma .
$$

Note that the value 0.5 of adhesive force marked with triangle in Figure 6 corresponds to exact JKR solution for the case of circular contact with $R_{1} / R_{2}=1$. The empirical equation (8), approximates this value for $R_{1} / R_{2}=1$ with 0.4976 .

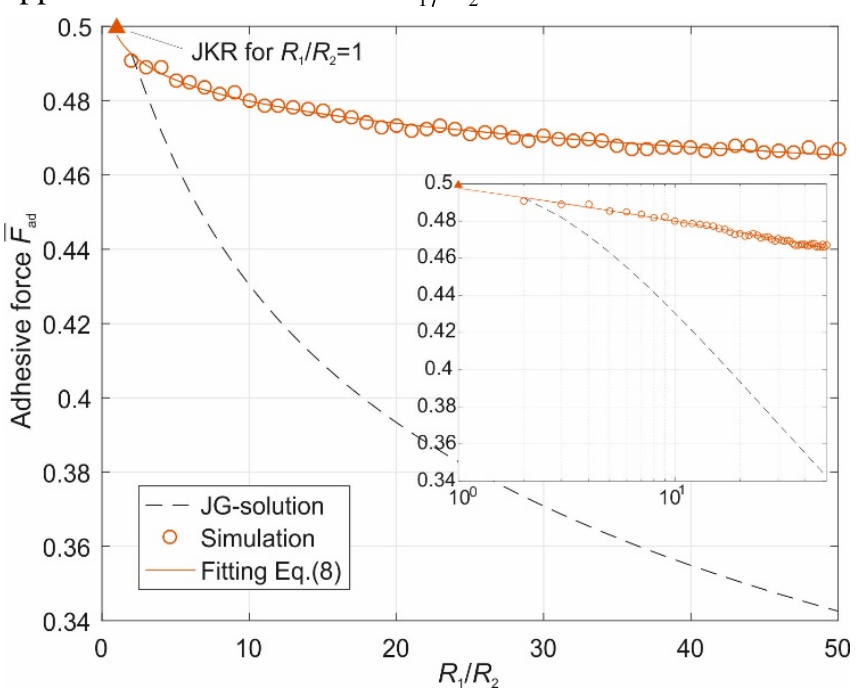

Figure 6 Dependence of adhesive force on the ratio $R_{1} / R_{2}$. The circles are numerical results, solid line the analytical expression (8) and dashed line the JG solution for the minimal pull-off force. The triangle corresponds to the JKR solution for the circular case.

\section{Discussion}

Contacts with variable ratio $R_{1} / R_{2}$ can be easily realized experimentally by pressing two identical circular cylinders against each other. If the axes of cylinders are perpendicular to each other, then $R_{1} / R_{2}=1$. By changing the angle between the axes, one can continually change this ratio. For example, the ratio $R_{1} / R_{2}=50$ is achieved at the angle of $16.1^{\circ}$. In the experimental study [6], the adhesive forces of two crossed cylinders with the identical radii $0.5 \mathrm{~mm}$ at different skew angles are provided. Using the same material and system parameter we simulated these pull-off processes with the equivalent ellipsoids. The comparison for adhesive forces for different angles are shown in Figure 7 together with the JGapproximation (solid line) and numerical results obtained in [10] where the surface interaction was assumed to be due to Lennard-Jones potential. It is seen that the absolute value of the force of adhesion according to the JG-solution is smaller than numerical and experimental results. In the numerical study [10], similar behavior including contact shape for contact between two crossed cylinders has been found for the large Tabor parameter $\mu=2.2$ (approaching the JKR-limit), and the force-distance curves are close to our numerical results.

Observing the adhesive force in Figure 6, it is seen that the absolute value of $\bar{F}_{a d}$ decreases not very much with increasing the ratio $R_{1} / R_{2}$ (by $7.4 \%$ for a large $R_{1} / R_{2}=50$ ). Thus, in the first approximation one could completely neglect this dependence and consider the corresponding dimensionless force of adhesion as constant and equal to 0.5 . In dimensional form, this means that the force of adhesion is given by the equation $F_{0}=3 / 2 \cdot \pi R_{e} \Delta \gamma$ with $R_{e}=\sqrt{R_{1} R_{2}}$. Thus, as a first approximation, the simplest approximation based on the concept of an equivalent circle is a good choice.

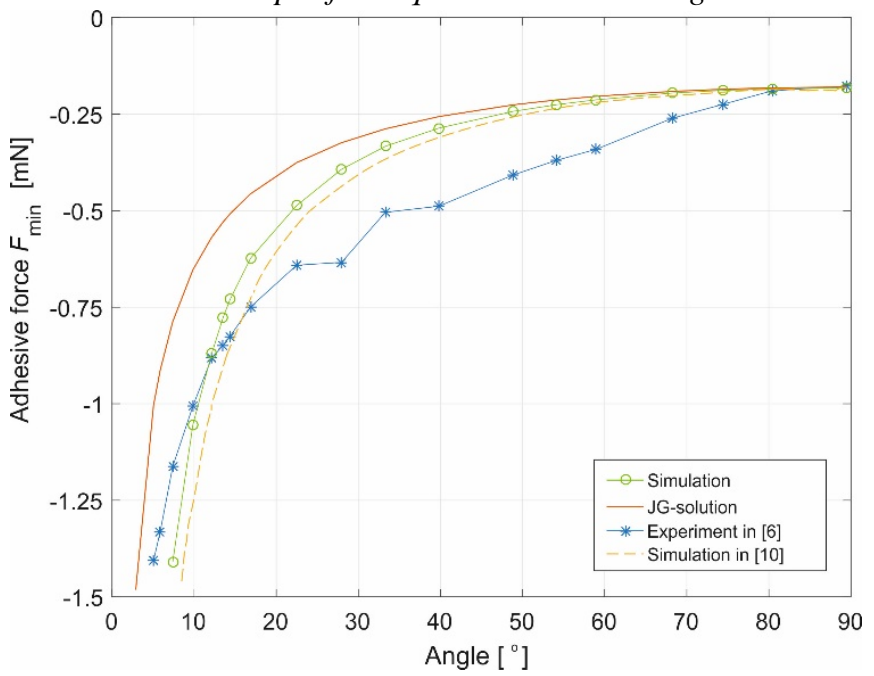

Figure 7 Comparison of adhesive force of JG-theory, experiment and simulations for equivalent contacts between two crossed identical cylinders by changing the angle between the axes.

\section{Conclusion}

We re-visited the old problem of adhesive contact of an ellipsoid with an elastic half space (or, equivalently, two crossed cylinders), which was initially approximately solved by Johnson and Greenwood. We used the "numerically exact" procedure based on the adhesive boundary element method. The force-displacement, force-mean contact radius as well as the maximal pull-off force were obtained and corresponding analytical approximations of numerical solutions were provided and compared with those of Johnson and Greenwood. The shape of contact area was found to be not exactly elliptical even for smaller ratio of principle radii of curvature $R_{1} / R_{2}$. The normalized adhesive force decreases with ratio $R_{1} / R_{2}$ but not as strongly as predicted by JGsolution. The simplest approximation based on replacing the elliptical contact by an equivalent circle contact with an 
effective radius of curvature $R_{e}=\sqrt{R_{1} R_{2}}$ gives already an acceptable precision even for relatively large values of the ratio $R_{1} / R_{2}$.

\section{Acknowledgements}

This work was partially funded by the German Research Society (DFG PO 810-55-1).

\section{References}

[1] Johnson K L, Kendall K and Roberts A D 1971 Surface energy and the contact of elastic solids Proc. R. Soc. London A 324 301-13

[2] Derjaguin B V, Muller V M and Toporov Y 1975 Effect of contact deformations on the adhesion of particles J. Colloid Interface Sci. 53 314-26

[3] Tabor D 1977 The hardness of solids J. Colloid Interface Sci. 58 145-79

[4] Maugis D 1992 Adhesion of spheres: The JKR-DMT transition using a Dugdale model J. Colloid Interface Sci. 150 243-69

[5] Johnson K L and Greenwood J A 2005 An approximate JKR theory for elliptical contacts J. Phys. D. Appl. Phys. 38 10426

[6] Sümer B, Onal C D, Aksak B and Sitti M 2010 An experimental analysis of elliptical adhesive contact J. Appl. Phys. 107 0-7

[7] Zini N H M, de Rooij M B, Bazr Afshan Fadafan M, Ismail N and Schipper D J 2018 Extending the Double-Hertz Model to Allow Modeling of an Adhesive Elliptical Contact Tribol. Lett. 66 1-13

[8] Greenwood J A 2018 On "extending the Double-Hertz Model to Allow Modeling of an Adhesive Elliptical Contact” Tribol. Lett. 66:88 1-3

[9] Wu J J 2006 Numerical analyses on elliptical adhesive contact J. Phys. D. Appl. Phys. 39 1899-907

[10] Jin C, Jagota A and Hui C-Y 2011 An easy-to-implement numerical simulation method for adhesive contact problems involving asymmetric adhesive contact J. Phys. D. Appl. Phys. 44405303

[11] Pohrt R and Popov V L 2015 Adhesive contact simulation of elastic solids using local mesh-dependent detachment criterion in boundary elements method Facta Univ. Ser. Mech. Eng. 13 3-10

[12] Argatov I, Li Q, Pohrt R and Popov V L 2016 JohnsonKendall-Roberts adhesive contact for a toroidal indenter Proc. R. Soc. London A Math. Phys. Eng. Sci. 472

[13] Li Q, Argatov I and Popov V L 2018 Onset of detachment in adhesive contact of an elastic half-space and flat-ended punches with non-circular shape : analytic estimates and comparison with numeric analysis J. Phys. D. Appl. Phys. 145601

[14] Popov V L, Pohrt R and Li Q 2017 Strength of adhesive contacts: Influence of contact geometry and material gradients Friction 5 308-325

[15] Lubrecht A A and Venner C H 2000 Multi-Level Methods in Lubrication Elsevier Science
[16] Pohrt R and Li Q 2014 Complete Boundary Element Formulation for Normal and Tangential Contact Problems Phys. Mesomech. 17, 334-340.

[17] Polonsky I A and Keer L M. 1999 A numerical method for solving rough contact problems based on the multi-level multi-summation and conjugate gradient techniques Wear $\mathbf{2 3 1}$ 206-219.

[18] Putignano C, Afferrante L, Carbone G and Demelio G A 2012 new efficient numerical method for contact mechanics of rough surfaces Int. J. Solids Struct. 49 338-343. 